\title{
Practicality of Wound Detection Using Smartphone Measure App
}

\author{
Chun-I FANG ${ }^{\mathrm{a}, \mathrm{b}, 1}$, Tseng-Tzu WU ${ }^{\mathrm{a}, \mathrm{b}}$, Chen-Yi CHU ${ }^{\mathrm{b}, \mathrm{c}}$ and Yen-Chiao LU ${ }^{\mathrm{b}}$ \\ ${ }^{a}$ Department of Intrathoracic Surgical, Chung Shan Medical University Hospital \\ ${ }^{\mathrm{b}}$ School of Nursing, Chung Shan Medical University Taichung, Taiwan \\ ${ }^{\mathrm{c}}$ Department of Hemodialysis Room, Chung Shan Medical University Hospital
}

\begin{abstract}
The record of the pressure sore with photo needs to be measurable. We compare the time consumption of wound assessment ruler and measure application of smartphone and the satisfaction of the users. The time needed is 20 and 35 seconds for the ruler and the application on average. But the satisfaction is better for application for its convenience, less infection, and the accuracy of measurements.
\end{abstract}

Keywords. Wound measurement, nursing information, smartphone application

\section{Introduction}

The delayed treatment of chronic wounds would result in severe infection, which consequences in increasing the usage of medical resources and worsening life quality for the patients. Therefore, the chronic wound is always an important care issue [1]. Wound measuring is important in monitoring the process of healing and evaluating the effect of the treatment. With the widespread of smartphones, the public can utilize the built-in software in smartphones to obtain reliable and objective images and parameters of the wounds which helps to evaluate and treat the wounds better. It also provides novel ways to improve medical services [2,3].

\section{Background and Purpose}

In recent years, there are many medical disputes caused by the family members' dissatisfaction of patients' pressure sore. However, From January to August 2019, a total of 13 pressure sore occurred in the general ward of a medical center in central Taiwan, with an incidence rate of $0.05 \%$; while our unit occurred three cases with an average incidence rate of $0.03 \%$. As a result, the medical quality control requires nurses use photographs and written notes to document pressure sore from the year of 2019. However, the investigators found that the majority of the nurses didn't use the wound assessment ruler when taking photos. Therefore, the size of the wound was not recorded well. As a result, this study will inspect whether using a proposed smartphone app to measure wound status would reduce the nurses' documentation burden.

\footnotetext{
${ }^{1}$ Corresponding Author, Chun-I Fang; E-mail: gemmy518@gmail.com.
} 


\section{Method}

This study aimed to compare the total documentation time and accuracy using the smartphone app and the wound assessment ruler. Questionnaires were then filled to see how the users evaluate the wound assessment ruler and the smartphone app. There are seven questions, use the 5 point Likert Scale questionnaires and that dividing the subjects judgment results into five categories: very disagree, disagree, No opinion, agree, very agree.

\section{Result}

The deviation after comparing the results of the two methods is 0.5 to $1 \mathrm{~cm}$. As shown in Figure 1. The result of this study showed an average of 20 seconds and 35 seconds for wound assessment ruler and smartphone app accordingly for 20 wounds. 28 registered professional nurses in this unit completed the questionnaire for satisfaction. $17.9 \%$ think the ruler is inconvenient, for it may not be taken while recording, and the contact of the ruler to the wound may cause infection. All 28 users were rather to use the measure application because that tool was always ready in hand, less treatment interrupting, and no need to remember the size of the wound, therefore, and increasing the accuracy of documentation of wound size.

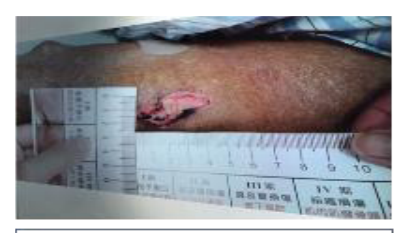

$2.5 \times 4 \mathrm{~cm}$

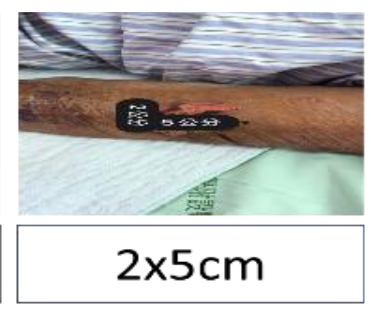

$2 \times 5 \mathrm{~cm}$

Figure 1. Wound size.

\section{Conclusions}

The difference between the two methods' time spending may due to: (1) Using the measure application of smartphone requires delicate finger pointing on a small screen, patients' movement can result in an error of the measurement, and the procedure restarted, which may lengthen the time. (2) The crews have 8 years of experience in the work, and therefore usually will not forget to take the wound assessment ruler, which results in no incidents that need to interrupt the treatment. Therefore, using the measure application that the number of tools needed was reduced, and it might reduce the risk of wound infection; no need to remember the size of the wound, therefore, increased accuracy toward documenting the size of the wound. If the users can directly upload the size and picture of the wound to the Hospital Information System when adding a builtin measure application to the smartphone, it can reduce the documentation time and there is no risk of wound infection since it makes no contact with the wound. 


\section{References}

[1] Lo SF, Hu WY. The principles of chronic wound assessment and measurement. The Journal of Nursing. 2006;54: 62-67.

[2] Chao E, Meenan CK, Ferris LK. Smartphone-based applications for skin monitoring and melanoma detection. Dermatol Clin. 2017; 35: 551-557.

[3] Wang SC, Anderson JA, Evans R et al. Point-of-care wound visioning technology: Reproducibility and accuracy of a wound measurement app. PLoS One. 2017; 12. Retrieved from https://doi.org/10.1371/journal.pone.0183139. 